\title{
Surfactant-controlled composition and crystal structure of manganese(II) sulfide nanocrystals prepared by solvothermal synthesis
}

\author{
Elena Capetti ${ }^{1}$, Anna M. Ferretti ${ }^{1}$, Vladimiro Dal Santo ${ }^{2}$ and Alessandro Ponti ${ }^{* 1, \S}$
}

\author{
Full Research Paper \\ Address: \\ ${ }^{1}$ Laboratorio di Nanotecnologie, Istituto di Scienze e Tecnologie \\ Molecolari, Consiglio Nazionale delle Ricerche, via G. Fantoli 16/15, \\ 20138 Milano, Italy, and ${ }^{2}$ Istituto di Scienze e Tecnologie Molecolari, \\ Consiglio Nazionale delle Ricerche, via C. Golgi 19, 20133 Milano, \\ Italy \\ Email: \\ Alessandro Ponti ${ }^{*}$ - alessandro.ponti@istm.cnr.it \\ * Corresponding author \\ § Tel.: +39025031 4280, Fax: +390250313927 \\ Keywords: \\ manganese oxide; manganese sulfide; nanocrystal; polymorphism \\ control; solvothermal synthesis; sulfur; surfactant \\ Beilstein J. Nanotechnol. 2015, 6, 2319-2329. \\ doi:10.3762/bjnano.6.238 \\ Received: 09 October 2015 \\ Accepted: 18 November 2015 \\ Published: 07 December 2015 \\ Associate Editor: J. J. Schneider \\ (c) 2015 Capetti et al; licensee Beilstein-Institut. \\ License and terms: see end of document.
}

\begin{abstract}
We investigated how the outcome of the solvothermal synthesis of manganese(II) sulfide (MnS) nanocrystals (NCs) is affected by the type and amount of long chain surfactant present in the reaction mixture. Prompted by a previous observation that a larger than stoichiometric amount of sulfur is required [Puglisi, A.; Mondini, S.; Cenedese, S.; Ferretti, A. M.; Santo, N.; Ponti A. Chem. Mater. 2010, 22, 2804-2813], we carried out a wide set of reactions using $\mathrm{Mn}(\mathrm{II})$ carboxylates and $\mathrm{Mn}_{2}(\mathrm{CO})_{10}$ as precursors with varying amounts of sulfur and carboxylic acid. MnS NCs were obtained provided that the $\mathrm{S} / \mathrm{Mn}$ ratio was larger than the $\mathrm{L} / \mathrm{Mn}$ ratio, otherwise $\mathrm{MnO}$ NCs were produced. Since $\mathrm{MnS}$ can crystallize in three distinct phases (rock salt $\alpha$-MnS, zincblende $\beta$-MnS, and wurtzite $\gamma-\mathrm{MnS}$ ), we also investigated whether the surfactant affected the NC polymorphism. We found that MnS polymorphism can be controlled by appropriate selection of the surfactant. $\gamma$-MnS nanocrystals formed when a 1:2 mixture of long chain carboxylic acid and amine was used, irrespective of the presence of carboxylic acid as a free surfactant or ligand in the metal precursor. When we used a single surfactant (carboxylic acid, alcohol, thiol, amine), $\alpha$-MnS nanocrystals were obtained. The peculiar role of the amine seems to be related to its basicity. The nanocrystals were characterized by TEM and electron diffraction; ATR-FTIR spectroscopy provided information about the surfactants adsorbed on the NCs.
\end{abstract}

\section{Introduction}

Manganese(II) sulfide (MnS) is a wide bandgap $\left(E_{\mathrm{g}} \approx 3 \mathrm{eV}\right)$ [1], p-type, antiferromagnetic semiconductor that crystallizes in three distinct phases: cubic $\alpha-\mathrm{MnS}$ (rock salt), cubic $\beta-\mathrm{MnS}$ (zincblende), and hexagonal $\gamma$-MnS (wurtzite). In $\alpha-\mathrm{MnS}$, sulfide anions form an fcc lattice and manganese cations fill all of the octahedral voids. $\beta-\mathrm{MnS}$ also has an fcc lattice of $\mathrm{S}^{2-}$ 
ions but the $\mathrm{Mn}^{2+}$ ions occupy half of the tetrahedral voids. In $\gamma$-MnS, the $\mathrm{S}^{2-}$ ions form an hep lattice and the $\mathrm{Mn}^{2+}$ ions again occupy half of the tetrahedral voids. $\alpha-\mathrm{MnS}$ is the thermodynamically stable form and is found in manganese ore beds as the mineral alabandite, which has been known since the early 19th century [2]. More recently, naturally occurring minerals with $\beta$ - and $\gamma$-MnS structure have been described. Rambergite $(\gamma-\mathrm{MnS})$ was discovered in 1996 in Sweden [3]. In 2012, extraterrestrial browneite $(\beta-\mathrm{MnS})$ was found in a meteorite collected in Poland [4]. All three forms of MnS display antiferromagnetic order [5] with a Néel temperature of $\approx 80 \mathrm{~K}$ $(\gamma-\mathrm{MnS}), \approx 100 \mathrm{~K}(\beta-\mathrm{MnS})$, and $154 \mathrm{~K}(\alpha-\mathrm{MnS})[6]$. The interesting physical properties and the rich polymorphism prompted research on $\mathrm{MnS}$ nanocrystals (NCs) in view of applications as photoluminescent components [7], photoreduction catalysts [8], anode materials in lithium-ion batteries [9], and supercapacitor materials [10].

Although MnS NCs have been synthesized by different methods, including chemical vapor deposition [11-13] and hydrothermal [14-16] methods, here we focus on NCs synthesized by solvothermal methods, which usually allow more experimental flexibility and improved NC control. Whereas reports on the solvothermal synthesis of $\beta-\mathrm{MnS} \mathrm{NCs}$ are rare [17], several syntheses of $\alpha-\mathrm{MnS}$ [18-27] and $\gamma-\mathrm{MnS}$ [19,22,2629] NCs have been described.

Diverse reagents were used to introduce sulfur in the reaction mixture: sulfur-containing Mn complexes [19-21,27], organic (thiourea [16], thioacetamide [26], bistrimethylsilylsulfide [18], dodecanethiol [30]) and inorganic (sulfur [22-24,28], ammonium sulfide [29]) compounds. The S/Mn molar ratio varied greatly, ranging from (1/3): 1 to $4: 1$, but in most cases $\mathrm{S} / \mathrm{Mn} \geq 1$. Use of $\mathrm{S} / \mathrm{Mn}<1$ was successful when carboxylic acids were not present in the reaction mixture. In a previous investigation of the solvothermal synthesis of MnS NCs from Mn(II) oleates [23], it was shown that in the absence of free surfactants, an excess of sulfur $(\mathrm{S} / \mathrm{Mn} \geq 2)$ is needed to avoid the formation of $\mathrm{MnO}$ along with (or in place of) MnS NCs. We further pursued this investigation aiming at finding the sulfur concentration required to produce $\mathrm{MnS}$ NCs when different Mn precursors are used and free surfactants are added.

In the majority of literature reports that the synthesis of $\mathrm{MnS}$ NCs leads to structurally pure products, that is, $\alpha-, \beta$ - or $\gamma-\mathrm{MnS}$ NCs. Of course, this is advantageous as different crystal phases display different physical properties. However, in contrast to size and shape control, relatively little attention has been paid to the factors controlling the crystal structure of MnS NCs. The reaction temperature is of course a key physical parameter since high temperature usually favors achievement of chemical equi- librium and the most stable phase. It was early described that heating manganese(II) diethyldithiocarbamate in hexadecylamine at low temperature $\left(120-150{ }^{\circ} \mathrm{C}\right)$ gave multipods comprising $\gamma-\mathrm{MnS}$ arms stemming from a $\beta-\mathrm{MnS}$ core, whereas $30 \mathrm{~nm} \alpha-\mathrm{MnS}$ cubes were obtained at $180{ }^{\circ} \mathrm{C}$ [19]. A more detailed investigation gave similar results for the reaction of manganese(II) chloride and thioacetamide in oleylamine, confirming the key role of temperature [26]. These authors also described the $\beta \rightarrow \alpha$ and $\gamma \rightarrow \alpha$ phase transformation of MnS NCs subject to high pressure. The formation of $\alpha$ - vs $\gamma-\mathrm{MnS}$ NCs was also shown to depend on the heating rate $\left(15^{\circ} \mathrm{C} / \mathrm{min}\right.$ : $\alpha$-MnS; 25 and $35{ }^{\circ} \mathrm{C} / \mathrm{min}: \gamma$-MnS) when manganese(II) diethyldithiocarbamate was heated in octadecene at $320{ }^{\circ} \mathrm{C}$ in the presence of a large excess of both oleic acid and oleylamine (100:1 with respect to $\mathrm{Mn}$ ) [27].

Polymorphism control could also be achieved by chemical means. By reacting manganese(II) chloride and thiourea in an autoclave $\left(T=190^{\circ} \mathrm{C}\right)$ for $12 \mathrm{~h}, 30 \mathrm{~nm} \alpha$-MnS NCs were prepared using water as a solvent, whereas when the solvent was benzene, $\gamma$-MnS rods $(d=40-100 \mathrm{~nm}, l=250-700 \mathrm{~nm})$ were obtained [16]. The reaction of manganese(II) nitrate with elemental $\mathrm{S}$ in octadecylamine at $200{ }^{\circ} \mathrm{C}$ gave $50 \mathrm{~nm} \alpha-\mathrm{MnS}$ hexagons at high $\mathrm{S}$ concentration, whereas $\gamma$-MnS rods $(d \approx 50 \mathrm{~nm})$ resulted at low $\mathrm{S}$ concentration [22]. The hydrothermal reaction of manganese(II) chloride with sodium sulfide at $180{ }^{\circ} \mathrm{C}$ yielded $200 \mathrm{~nm} \alpha-\mathrm{MnS}$ octahedral NCs but $\gamma$-MnS rods $(d=200-300 \mathrm{~nm}, l=1.0-1.5 \mu \mathrm{m})$ were obtained when a large excess of hydrazine was added [15]. In summary, the control of the crystal structure of MnS NCs was achieved by varying the solvent, the amount of sulfur, or adding a reducing agent such as hydrazine. The effect of surfactant type on the crystal phase of MnS NCs has not yet been studied, to the best of our knowledge.

In this paper, we report on the control of the composition and crystal structure of MnS NCs obtained by solvothermal decomposition of sulfur-free manganese precursors in the presence of elemental sulfur and long chain surfactants. In particular, we will focus on two issues: (1) the effect of surfactant (L) and sulfur (S) on the formation of either MnO or MnS NCs and (2) the effect of the surfactant on the polymorphism of $\mathrm{MnS}$ NCs. We will show that the formation of MnS vs MnO NCs depends, in a nontrivial manner, on both $\mathrm{S} / \mathrm{Mn}$ and $\mathrm{L} / \mathrm{Mn}$ molar ratios. Additionally, we will show that the formation of $\alpha-\mathrm{MnS}$ vs $\gamma$-MnS NCs can be controlled by selecting the surfactant(s) present in the reaction mixture. We unexpectedly found that $\alpha-\mathrm{MnS}$ NCs are obtained when a single surfactant is present, irrespective of its polar end group, whereas $\gamma$-MnS NCs form when a mixture of long chain amine and carboxylic acid is used. 


\section{Results and Discussion}

In this paper we focus on how the composition and crystal structure of NCs are affected by (1) the choice of surfactant and (2) the concentration of sulfur and surfactant. In order to study the effect of the reactant type and concentration on the composition and polymorphism of the resulting NCs, all reactions were carried out using a common synthetic procedure, that is, heating to $320^{\circ} \mathrm{C}$ (heating rate $=10^{\circ} \mathrm{C} / \mathrm{min}$ ) in an octadecene solution of $\mathrm{Mn}$ precursor, $\mathrm{S}$, and (possibly) surfactant, and finally, ageing for $1 \mathrm{~h}$ before cooling and isolating the resulting NCs.

\section{MnO vs MnS: The role of sulfur and surfactant}

As anticipated in the Introduction, it was observed that when $\mathrm{MnOl}(\mathrm{OH})$ or $\mathrm{MnOl}_{2}$ (Ol - oleate) was decomposed in octadecene in the absence of any surfactant, a stoichiometric amount of sulfur led to a mixture of $\mathrm{MnO}$ and $\alpha-\mathrm{MnS} \mathrm{NCs}$ (pure batches of $\alpha-M n S$ NCs could be achieved using a S/Mn molar ratio $\geq 2$ whereas pure $\mathrm{MnO}$ NCs were obtained using $\mathrm{S} / \mathrm{Mn} \leq 0.6)$ [23]. To gain a deeper insight, we carried out several reactions where a manganese precursor was thermally decomposed in octadecene containing varying amounts of sulfur and free surfactant (L).

As manganese precursors we used manganese(II) carboxylates $\left(\mathrm{Mn}(\mathrm{OH}) \mathrm{Ol}, \mathrm{MnOl}_{2}\right.$, and $\mathrm{MnSt}_{2}$; abbreviations of chemical names can be found in the Experimental section) and an inorganic compound, $\mathrm{Mn}_{2}(\mathrm{CO})_{10}$, which cannot release carboxylic acid upon decomposition. Transmission electron microscopy (TEM) images and electron diffraction (ED) patterns of representative NCs can be found in Figure 1. All ED patterns could be assigned to $\mathrm{MnO}$ or $\alpha-\mathrm{MnS} \mathrm{NCs}$ (or a mixture of both). The rock salt structure of MnS NCs was confirmed by HRTEM: Figure 2a displays lattice fringes separated by $0.258 \mathrm{~nm}$ that correspond to the $\{200\}$ planes of the $\alpha-\mathrm{MnS}$ structure. The geometric phase analysis (GPA) [31] showed that the NCs are single crystallites, almost free from lattice defects.

The chemical composition ( $\mathrm{MnO}$ vs $\mathrm{MnS}$ ) of the resulting NCs depended on the $\mathrm{L} / \mathrm{Mn}$ and $\mathrm{S} / \mathrm{Mn}$ molar ratios, as graphically summarized in Figure 3. Open (solid) squares indicate that $\mathrm{MnO}(\mathrm{MnS}) \mathrm{NCs}$ were obtained, and half-filled squares are used to represent a $\mathrm{MnO} / \mathrm{MnS} \mathrm{NC}$ mixture. The reactions carried out with no free surfactant correspond to data on the vertical axis of Figure 3. These results confirmed the previous conclusions about $\mathrm{Mn}(\mathrm{OH}) \mathrm{Ol}$ or $\mathrm{MnOl}_{2}$, in particular that a mixture of $\mathrm{MnO}$ and $\alpha-\mathrm{MnS} \mathrm{NCs}$ is obtained at a stoichiometric $\mathrm{S} / \mathrm{Mn}=1: 1$ ratio. The electron diffraction results gave no evidence that $\mathrm{MnO}_{x} \mathrm{~S}_{1-x} \mathrm{NCs}$ formed despite that both $\mathrm{MnO}$ and $\alpha-\mathrm{MnS}$ have a rock salt structure. We investigated a wide range of ageing times (30-180 $\mathrm{min}$ ) and such a NC mixture was

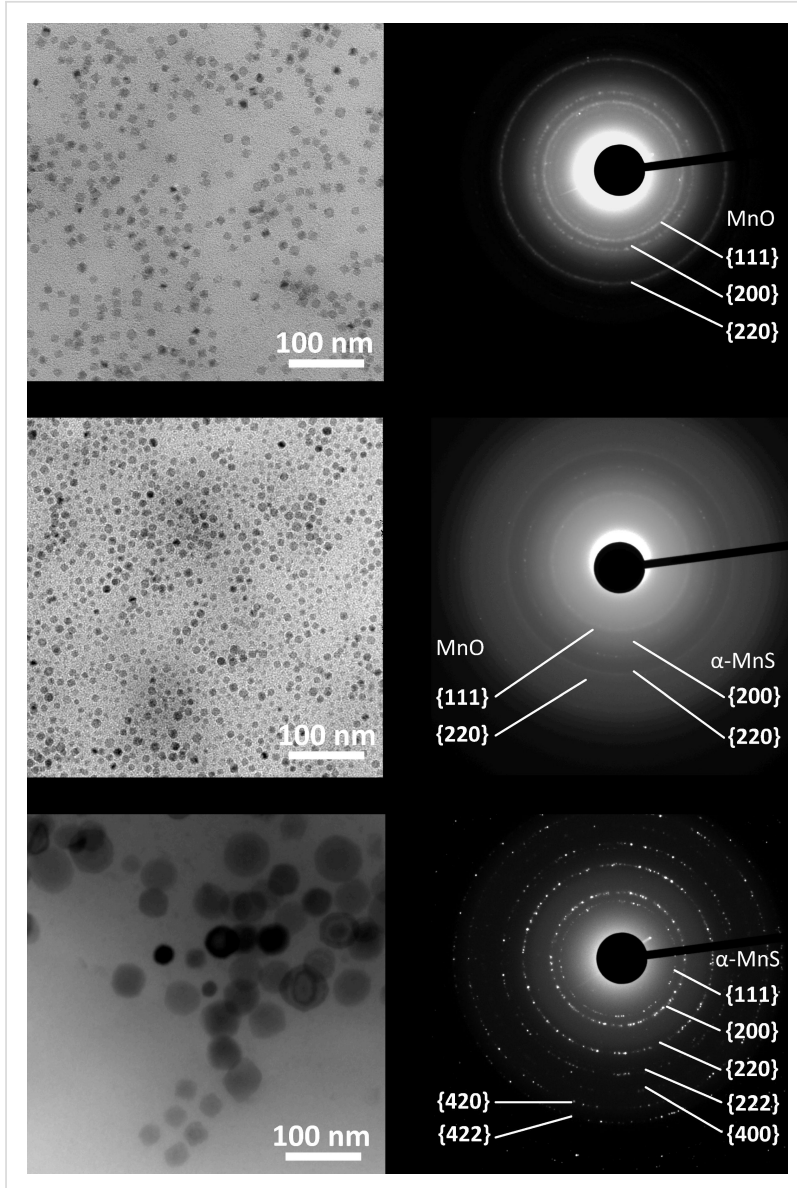

Figure 1: TEM images (left) and electron diffraction patterns (right) of $\mathrm{NCs}$ obtained by high temperature decomposition of $\mathrm{Mn}_{2}(\mathrm{CO})_{10}$ with $\mathrm{StAc} / \mathrm{Mn}=2$ and varying amounts of sulfur. (a) $\mathrm{S} / \mathrm{Mn}=1, \mathrm{MnO} N \mathrm{NC}$;

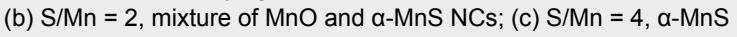
NCs.

obtained in all cases, showing that interconversion of $\mathrm{MnO}$ and $\alpha-\mathrm{MnS}$ NCs during ageing did not occur. Both of these findings are probably related to the largely different ionic radius of hexacoordinated $\mathrm{O}^{2-}(0.126 \mathrm{~nm})$ and $\mathrm{S}^{2-}(0.170 \mathrm{~nm})$ [32].

We also carried out reactions where a free surfactant (L) was added to the reaction mixture. In the case of $\mathrm{Mn}_{2}(\mathrm{CO})_{10}$, we added free StAc. From Figure 3, one can see that we obtained a $\mathrm{NC}$ mixture when the molar amount of $\mathrm{S}$ and $\mathrm{L}$ were equal. Pure MnO NCs were prepared when the amount of S was lower than that of L. Conversely, when the amount of S was higher than L, the reaction yielded pure $\alpha-M n S$ NCs. In the case of Mn carboxylate precursors, the free surfactant was the corresponding carboxylic acid, i.e., OlAc for $\mathrm{Mn}(\mathrm{OH}) \mathrm{Ol}$ or $\mathrm{MnOl}_{2}$ and $\mathrm{StAc}$ for $\mathrm{MnSt}_{2}$. We again found that, in general, pure $\alpha-\mathrm{MnS}$ NCs were obtained in sulfur-rich reactions and pure $\mathrm{MnO}$ NCs in surfactant-rich reactions. Reactions involving carboxylate precursors carried out using high S/Mn and L/Mn ratios often did not yield NCs. 


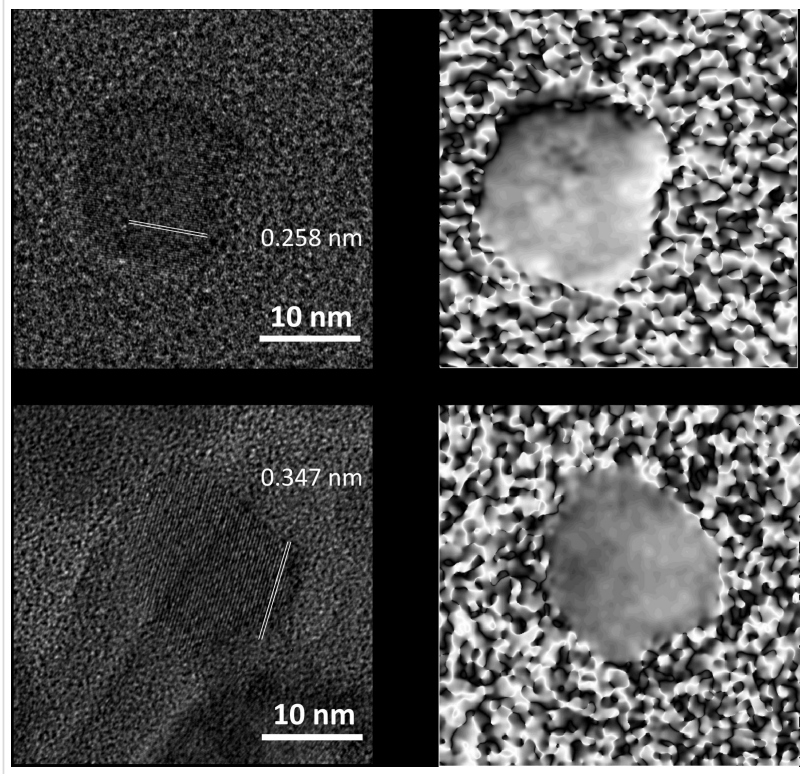

Figure 2: HRTEM images (left) and geometric phase analysis (GPA,

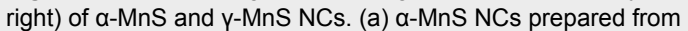
$\mathrm{Mn}_{2}(\mathrm{CO})_{10}$ in the presence of StAc (S/StAc/Mn = 2:4:1, where St $=$ stearic acid) displaying $0.258 \mathrm{~nm}$ lattice fringes corresponding to $\{200\}$ planes. (b) $\mathrm{Y}$-MnS NC prepared from $\mathrm{MnSt}_{2}$ in the presence of $\mathrm{HdAm}$ ( $\mathrm{S} / \mathrm{HdAm} / \mathrm{Mn}=2: 4: 1$, where $\mathrm{HdAm}=$ hexadecylamine) displaying $0.347 \mathrm{~nm}$ lattice fringes corresponding to $\{100\}$ planes.

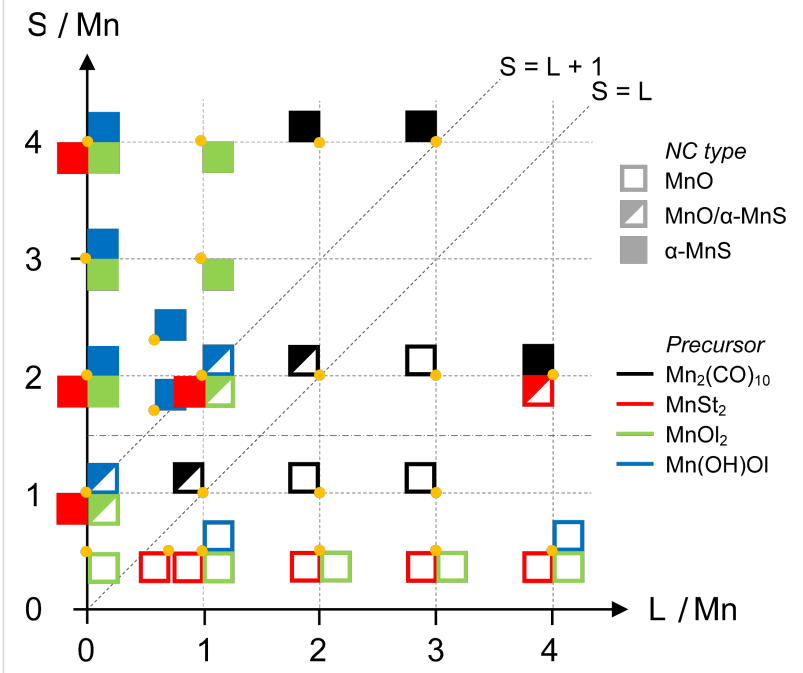

Figure 3: Pictorial representation of the NC outcome from the solvothermal synthesis involving the decomposition of different $\mathrm{Mn}$ precursors as a function of the L/Mn and S/Mn molar ratio. Each orange dot represents synthesis at the corresponding values of $L / M n$ and $S / M n$. One to four squares arranged about each dot represent the resulting NCs when using different Mn precursors. The type of NC is encoded as follows: open squares: MnO NCs; half-filled squares: mixture of $\mathrm{MnO}$ and $\alpha-\mathrm{MnS}$ NCs; solid squares: $\alpha-M n S$ NCs. The precursors are color coded as follows (counterclockwise from top left): black: $\mathrm{Mn}_{2}(\mathrm{CO})_{10}$, red: $\mathrm{MnSt}_{2}$, green: $\mathrm{MnOl}_{2}$, blue: $\mathrm{Mn}(\mathrm{OH}) \mathrm{Ol}$. The free surfactant, L, was StAc for $\mathrm{Mn}_{2}(\mathrm{CO})_{10}$ and $\mathrm{MnSt}_{2}$, and OIAc for $\mathrm{Mn}(\mathrm{OH}) \mathrm{Ol}$ and $\mathrm{MnOl}_{2}$, respectively.
On the whole, the resulting NC composition depends on sulfur and surfactant in a qualitatively simple way, which can be summarized as follows. When carboxylic acid is added as free surfactant, the amount of sulfur needed to produce MnS NCs increases. The plot in Figure 3 can be divided into two regions corresponding to $\mathrm{MnO}$ (bottom right) and $\alpha-\mathrm{MnS}$ (top left) with a boundary running diagonally in an approximately linear way. Using $\mathrm{Mn}_{2}(\mathrm{CO})_{10}$ as a precursor, it bisects the plot $(\mathrm{S} / \mathrm{L}=1: 1)$. When $\mathrm{Mn}(\mathrm{OH}) \mathrm{Ol}$ or $\mathrm{MnOl}_{2}$ are used, the boundary is shifted towards the left side by about one unit $(\mathrm{S} /(\mathrm{L}+1)=1: 1)$, suggesting that the oleate within the precursor plays a role in favoring the formation of $\mathrm{MnO} \mathrm{NCs}$. In this regard, $\mathrm{MnSt}_{2}$ is less efficient in that pure $\alpha-\mathrm{MnS} \mathrm{NCs}$ formed under conditions $(\mathrm{L} / \mathrm{Mn}=0: 1, \mathrm{~S} / \mathrm{Mn}=1: 1$ and $\mathrm{L} / \mathrm{Mn}=1: 1, \mathrm{~S} / \mathrm{Mn}=2: 1)$ that led to $\alpha-\mathrm{MnS} / \mathrm{MnO} \mathrm{NC}$ mixtures with the Mn mono- and dioleate precursors.

It was previously shown that at $180^{\circ} \mathrm{C}$ elemental sulfur, which is present as cyclo-, catena- and polymeric species [33], reacts with 1 -octadecene to produce more reactive $\mathrm{H}_{2} \mathrm{~S}$ (yield $\approx 2 / 3$ ), which reacts with cadmium oleate to give CdS NCs [34]. It is reasonable to think that $\mathrm{H}_{2} \mathrm{~S}$ is the actual sulfur source in the present case, too. Whereas in the case of CdS it was established that the carboxylic acid did not react with $\mathrm{H}_{2} \mathrm{~S}$, in our case, the decreased sulfur availability at increased surfactant concentration suggests that reaction between $\mathrm{RCOOH}$ and $\mathrm{H}_{2} \mathrm{~S}$ occurs. A possible reaction, which has long been known [35], is the transformation of $\mathrm{RCOOH}$ into the corresponding thio acid $\mathrm{RC}(\mathrm{O}) \mathrm{SH}$ by $\mathrm{H}_{2} \mathrm{~S}$. The occurrence of this (or a similar) reaction would explain the observed behavior (see Figure 3) provided that the product does not appreciably react with the Mn precursor. This explanation would also agree with the slightly different behavior of StAc and OlAc. Since $\mathrm{MnSt}_{2}$ decomposes at a higher temperature $\left(310-360{ }^{\circ} \mathrm{C}\right)$ [36] than manganese(II) oleate $\left(250-320^{\circ} \mathrm{C}\right)$ [37], it can be argued that at the reaction temperature of $320^{\circ} \mathrm{C}, \mathrm{MnSt}_{2}$ decomposes and releases StAc more slowly and/or to a lesser extent than manganese(II) oleates so that more $\mathrm{H}_{2} \mathrm{~S}$ is available for the formation of MnS NCs. One may wonder why the reaction of $\mathrm{H}_{2} \mathrm{~S}$ with the carboxylic acid was not observed in the synthesis of CdS NCs. Our synthesis was carried out at a higher temperature $\left(320^{\circ} \mathrm{C}\right)$ and reagent concentration $([\mathrm{Mn}]=0.25 \mathrm{M},[\mathrm{S}]$ and $[\mathrm{L}]=0-1 \mathrm{M})$ than that of the $\mathrm{CdS} \mathrm{NCs}\left(180^{\circ} \mathrm{C},[\mathrm{Cd}]=\right.$ $0.02 \mathrm{M},[\mathrm{S}]=0.01 \mathrm{M},[\mathrm{L}]=0.24 \mathrm{M})$, which may speed up the reaction of $\mathrm{H}_{2} \mathrm{~S}$ with $\mathrm{RCOOH}$. Additionally, the possible catalytic activity of $\mathrm{Mn}(\mathrm{II})$, a $\mathrm{d}^{5}$ ion with many possible oxidation states in comparison to $\mathrm{d}^{10} \mathrm{Cd}(\mathrm{II})$, should not be overlooked.

A complete collection of the NC morphological data can be found in Supporting Information File 1. Here, it is sufficient to 
say that MnO NCs usually have an octahedral shape with size depending on the surfactant: it increases from $\mathrm{Mn}_{2}(\mathrm{CO})_{10}$ $(10-20 \mathrm{~nm})$ to $\mathrm{MnSt}_{2}$ (up to $30 \mathrm{~nm}$ ). $\alpha$-MnS NCs are larger (10-65 nm), more polydisperse (15-35\%) and have irregular (but in all cases convex) shape.

Recalling Figure 3, one can note that the reaction with $\mathrm{S} / \mathrm{Mn}=2: 1$ and $\mathrm{L} / \mathrm{Mn}=4: 1$ yielded $\alpha-\mathrm{MnS}$ NCs or a mixture of $\alpha-\mathrm{MnS}$ and $\mathrm{MnO} \mathrm{NCs}$ when using $\mathrm{Mn}_{2}(\mathrm{CO})_{10}$ or $\mathrm{MnSt}_{2}$, respectively. This outcome does not conform to the above view of a diagonal boundary separating $\alpha-\mathrm{MnS}$ and $\mathrm{MnO}$ regions. We therefore decided to further investigate the $\mathrm{S} / \mathrm{L} / \mathrm{Mn}=2: 4: 1$ reaction in more detail by selecting $\mathrm{L}$ from a wide set of long chain surfactants of different chemical nature.

\section{The influence of free surfactant on the crystal structure of MnS NCs}

In this subsection, we report on the synthetic outcome of the thermal decomposition of manganese(II) distearate $\left(\mathrm{MnSt}_{2}\right)$ carried out with the $\mathrm{S} / \mathrm{L} / \mathrm{Mn}$ molar ratio fixed at 2:4:1. The long chain surfactant, L, was selected from a set including carboxylic acid, amine, alcohol, and thiol surfactants. This set allowed us to understand how basicity, nucleophilicity and ability to undergo condensation reaction with stearic acid affect the structure directing ability of the surfactant. The crystal structure of the resulting NCs was determined by analyzing the electron diffraction patterns. Selected ED patterns and TEM images are displayed in Figure 4. The identification of the crystal structure and the crystalline quality of the MnS NCs was confirmed by HRTEM images (Figure 2b). The crystal structure of the resulting NCs is summarized in Table 1 along with the morphological parameters.

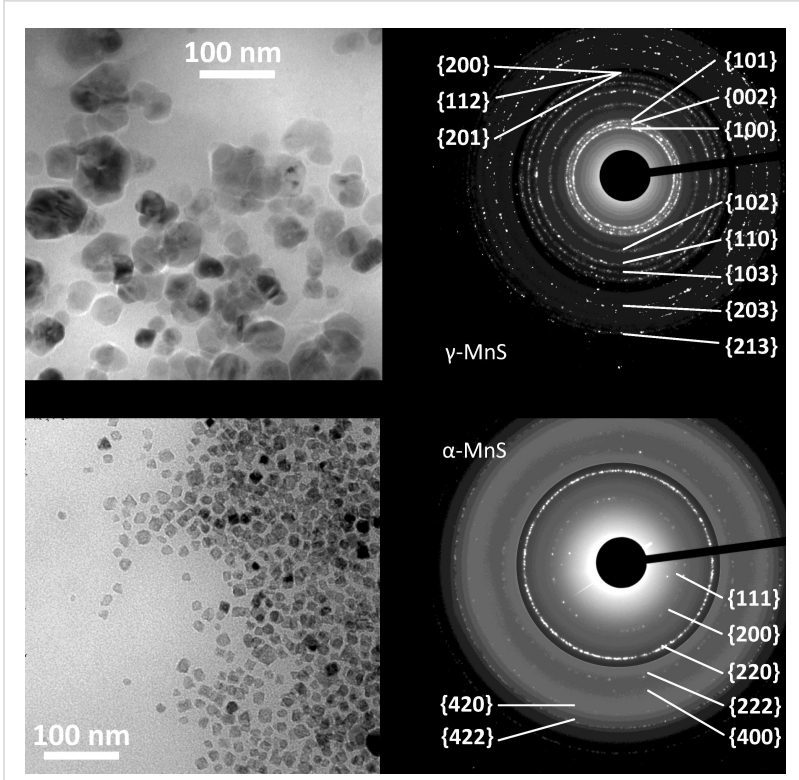

Figure 4: TEM images (left) and electron diffraction patterns (right) of $\mathrm{NCs}$ obtained by high temperature decomposition of $\mathrm{MnSt}_{2}$ with $\mathrm{S} / \mathrm{Mn}=2$ and $\mathrm{L} / \mathrm{Mn}=4$. (a) $\mathrm{y}-\mathrm{MnS}$ NCs synthesized using $\mathrm{L}=\mathrm{HdAm}$; (b) $\alpha-M n S$ NCs synthesized using L = DdTh (where DdTh = dodecanethiol). The contrast of the outer part of both diffraction patterns was digitally increased to show high-index rings.

It is clear from Table 1 that amines direct the reaction toward $\gamma$-MnS NCs while all other free surfactants (or the absence thereof) lead to $\alpha$-MnS NCs. This $\gamma$-directing effect, independent of the amine chain length and degree of saturation, seems to be related to the basicity of amines, which is highest within our surfactant set [38]. Nucleophilicity can be excluded since alkyl amines are less nucleophilic than sulfur compounds [39]. Similarly, the ability to undergo condensation reaction

Table 1: Properties of NCs synthesized by the thermal decomposition of $\mathrm{MnSt}_{2}$ with $\mathrm{S} / \mathrm{Mn}=2$ and $\mathrm{L} / \mathrm{Mn}=4$.

\begin{tabular}{|c|c|c|c|c|}
\hline L & NC type & Shape $^{a}$ & Size $(n m)^{b}$ & Std. dev. (nm) \\
\hline dodecylamine & $\mathrm{Y}-\mathrm{MnS}$ & IRC & $42 \times 29$ & $7 \times 11$ \\
\hline hexadecylamine & $\mathrm{Y}-\mathrm{MnS}$ & IRC & $55 \times 38$ & $15 \times 10$ \\
\hline octadecylamine & $\mathrm{Y}-\mathrm{MnS}$ & IRC & $55 \times 38$ & $12 \times 9$ \\
\hline oleylamine & $\mathrm{Y}-\mathrm{MnS}$ & IRC & $27 \times 20$ & $10 \times 8$ \\
\hline OIAm/DdTh ${ }^{c}$ & Y-MnS & IRC & $10-200$ & large \\
\hline none ${ }^{d}$ & a-MnS & $\begin{array}{l}\text { spherical } \\
\text { IRC }\end{array}$ & $\begin{array}{l}65 \\
12 \times 8\end{array}$ & $\begin{array}{l}10 \\
4 \times 2\end{array}$ \\
\hline oleylalcohol & a-MnS & IRC & $12 \times 8$ & $3 \times 2$ \\
\hline dodecanethiol & $\alpha-M n S$ & octahedral & 12 & 2 \\
\hline stearic acid ${ }^{\text {d,e }}$ & $\begin{array}{l}\alpha-\mathrm{MnS} \\
\mathrm{MnO}\end{array}$ & $\begin{array}{l}\text { spherical } \\
\text { IRC }\end{array}$ & $\begin{array}{l}7 \\
31 \times 23\end{array}$ & $\begin{array}{l}1 \\
4 \times 3\end{array}$ \\
\hline
\end{tabular}

${ }^{a} \mathrm{IRC}=$ irregular, rounded, convex shape; ${ }^{\mathrm{b}}$ Mean size, in the case of anisotropic NCs, the mean size of the major and minor axis are reported; 'Oleylamine/dodecanethiol mixture (1:1 mol/mol); ${ }^{d}$ Two morphological classes observed in TEM images (See Supporting Information File 1$)$; ${ }^{\text {e This }}$ sample also contains rare concave-shaped NCs (See Supporting Information File 1). 
with carboxylic acids can be excluded since oleyl alcohol could react with stearic acid to give the corresponding ester. This was observed when long chain, $\omega$-hydroxy carboxylic acids were employed in similar conditions [40]. Moreover, $\gamma-\mathrm{MnS}$ NCs were also obtained when the free surfactant was a $1: 1 \mathrm{~mol} / \mathrm{mol}$ mixture of OlAm and DdTh. This suggests that DdTh does not play a major role in the formation of MnS NCs, that is, it does not compete with $\mathrm{H}_{2} \mathrm{~S}$ as a sulfur source, despite that it has often been used to synthesize metal sulfide NCs [41].

The NC morphology is summarized in Table 1, and TEM images can be found in Figure 4 and in Supporting Information File 1. $\gamma$-MnS NCs have similar morphology: they are irregular, rounded, and convex (IRC) NCs with an aspect ratio of 1.4-1.5; the size slightly depends on the particular amine present. The O1Am/DdTh surfactant mixture instead gave broadly polydisperse $\gamma$-MnS NCs. $\alpha$-MnS NCs are in general smaller and have more regular shape than $\gamma$-MnS NCs. The octahedral shape of the $\alpha$-MnS NCs obtained using L $=$ DdTh is confirmed by the anomalous intensity of the electron diffraction rings (Figure $4 b$ ). Indeed, the outstanding $\{220\}$ ring is signature of the highly crystalline texture induced by the octahedral NC shape [23].

\section{Role of stearate ligand in the precursor molecule}

To understand the role that the stearate ligand presents in the precursor in the above reactions leading to $\gamma$-MnS NCs, we carried out synthetic experiments similar to those described in the previous subsection, employing $\mathrm{Mn}_{2}(\mathrm{CO})_{10}$ as a precursor and restricting ourselves to amines as a free surfactant. The main NC features are collected in Table 2, where TEM images and ED patterns of the $\mathrm{L}=\mathrm{HdAm}$ case can be found in Figure 5a (see also the Supporting Information File 1). Unexpectedly, we found that $\alpha-\mathrm{MnS}$ NCs were obtained in all cases, although a few $\gamma$-MnS NCs were intermixed with $\alpha-\mathrm{MnS}$ NCs only when OlAm was used. The $\gamma$-directing effect of amines observed with $\mathrm{MnSt}_{2}$ vanished when $\mathrm{Mn}_{2}(\mathrm{CO})_{10}$ was used,
Table 2: Properties of NCs synthesized by the thermal decomposition of $\mathrm{Mn}_{2}(\mathrm{CO})_{10}$ with $\mathrm{S} / \mathrm{Mn}=2$ and $\mathrm{L} / \mathrm{Mn}=4$.

\begin{tabular}{lllll} 
L & NC type & Shape $^{\mathrm{a}}$ & $\begin{array}{l}\text { Size } \\
(\mathrm{nm})\end{array}$ & $\begin{array}{l}\text { Std. dev. } \\
(\mathrm{nm})\end{array}$ \\
\hline oleylamine & $\begin{array}{l}\alpha-\mathrm{MnS} \\
(\mathrm{\gamma}-\mathrm{MnS})\end{array}$ & $\mathrm{IRC}$ & $27 \times 20$ & $9 \times 6$ \\
dodecylamine & $\alpha-M n S$ & $\mathrm{IRC}$ & $18 \times 14$ & $6 \times 4$ \\
hexadecylamine & $\alpha-M n S$ & IRC & $22 \times 16$ & $4 \times 3$ \\
octadecylamine & $\alpha-M n S$ & IRC & $20 \times 14$ & $4 \times 3$ \\
\hline
\end{tabular}

IRC = irregular, rounded, convex shape.

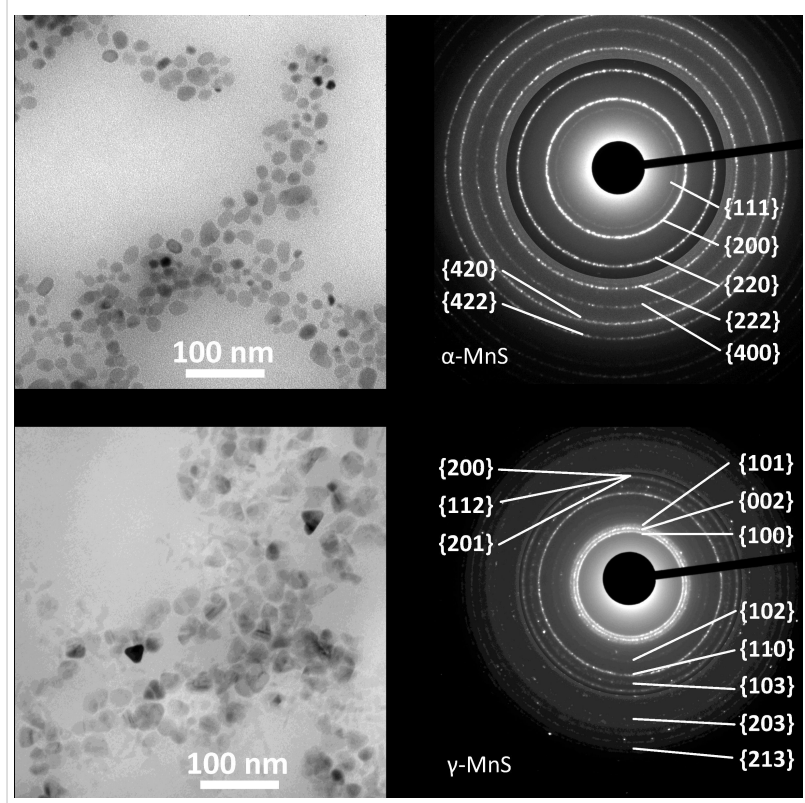

Figure 5: TEM images (left) and electron diffraction patterns (right) of NCs obtained by high temperature decomposition of $\mathrm{Mn}_{2}(\mathrm{CO})_{10}$ with $\mathrm{S} / \mathrm{Mn}=2$. (a) $\alpha-M n S$ NCs synthesized using $\operatorname{HdAm}(\mathrm{L} / \mathrm{Mn}=4)$;

(b) $y-M n S$ NCs synthesized using a mixture of StAc $\left(L_{\text {acid }} / \mathrm{Mn}=2: 1\right)$ and $\operatorname{HdAm}\left(\mathrm{L}_{\text {amine }} / \mathrm{Mn}=4\right)$. The contrast of the outer part of both diffraction patterns was digitally increased to show the high indexrings.

\begin{tabular}{|c|c|c|c|c|c|}
\hline $\mathrm{L}_{\text {acid }}$ & Lamine & NC type & Shape ${ }^{a}$ & Size $(\mathrm{nm})$ & Std. dev. (nm) \\
\hline stearic acid & oleylamine ${ }^{b}$ & $\alpha-M n S$ & spherical IRC & $\begin{array}{c}33 \\
15 \times 11\end{array}$ & $\begin{array}{c}6 \\
4 \times 3\end{array}$ \\
\hline stearic acid & dodecylamine & $\mathrm{y}-\mathrm{MnS}$ & IRC & $24 \times 18$ & $9 \times 7$ \\
\hline stearic acid & hexadecylamine & $\mathrm{y}-\mathrm{MnS}$ & IRC & $30 \times 21$ & $7 \times 5$ \\
\hline stearic acid & octadecylamine & Y-MnS & IRC & $35 \times 24$ & $10 \times 7$ \\
\hline oleic acid & hexadecylamine ${ }^{b}$ & Y-MnS & $\begin{array}{l}\text { spherical } \\
\text { spherical }\end{array}$ & $\begin{array}{l}11 \\
28\end{array}$ & $\begin{array}{l}2 \\
7\end{array}$ \\
\hline
\end{tabular}

a $\mathrm{IRC}=$ irregular, rounded, convex shape; ${ }^{\mathrm{b}}$ Two morphological classes observed in TEM images (See Supporting Information File 1). 
pointing at an important role of the stearate ligand in the polymorphism control. The NCs were smaller and had a lower aspect ratio than the corresponding ones prepared starting from $\mathrm{MnSt}_{2}$.

To gain more insight into the role of stearate, we carried out reactions where $\mathrm{Mn}_{2}(\mathrm{CO})_{10}$ was decomposed using a mixture of free surfactants including, as above, an amine $\left(\mathrm{L}_{\text {amine }} / \mathrm{Mn}=4: 1, \mathrm{~L}_{\text {amine }}=\mathrm{OlAm}, \mathrm{DdAm}, \mathrm{HdAm}\right.$, OdAm) and a carboxylic acid in $\mathrm{L}_{\mathrm{acid}} / \mathrm{Mn}=2: 1$ molar ratio $\left(\mathrm{L}_{\mathrm{acid}}=\mathrm{StAc}, \mathrm{OlAc}\right)$. The $\mathrm{S} / \mathrm{Mn}$ ratio was 2 , as before. These reactions mimicked those carried out using $\mathrm{MnSt}_{2}$ except that StAc is here present as a free surfactant and not as a ligand within the Mn precursor. The main features of the resulting NCs are collected in Table 3 and a TEM image and ED pattern of the $L_{\text {amine }}=$ HdAm case can be found in Figure $5 \mathrm{~b}$ (see also the Supporting Information File 1). We obtained $\gamma$-MnS NCs when saturated amines were used as a surfactant. This is evidence that both amine and stearic acid are required in the reaction mixture to direct the synthesis towards $\gamma$-MnS NCs. The irregularly shaped $\gamma$-MnS NCs were somewhat smaller than those prepared from $\mathrm{MnSt}_{2}$ and had a similar aspect ratio (1.35-1.45).

OlAm represented an exception in that $\alpha$-MnS NCs were obtained. This could depend on the presence of a double bond in the OlAm chain, which is the main structural difference between OlAm and the other amines. To investigate this issue, we performed a similar reaction using a mixture of OlAc and HdAm, which yielded $\gamma$-MnS NCs. Thus, the presence of a double bond in the free surfactant chain does not favor the formation of $\alpha$ - vs $\gamma$-MnS NCs. Considering that OlAm also showed a peculiar behavior in the reaction where StAc was absent (see Table 2), one might be tempted to ascribe these differences to the low purity of commercially available OlAm [42].

The observation that both OlAc/HdAm and StAc/(saturated amines) surfactant mixtures direct the synthetic outcome towards $\gamma$-MnS NCs supports the view that the formation of $\gamma$-MnS NCs is insensitive to the structural details of both surfactants.

In conclusion, the data presented herein are evidence that to prepare $\gamma$-MnS NCs from a solution of elemental sulfur and a manganese precursor in octadecene, it necessary that the reaction mixture comprises both amine and carboxylic acid surfactants, otherwise $\alpha$-MnS NCs are formed. Whether the carboxylic acid is present as free surfactant or as carboxylate ligand within the Mn precursor is irrelevant to the crystal structure of the resulting NCs.

\section{ATR-IR spectroscopy of MnS NCs}

The attenuated total reflectance infrared (ATR-IR) spectra of the NCs was recorded for several reactions (Tables 1-3) to investigate the organic species present on the NC surface at the end of the reaction. In particular, we analyzed NCs from reactions using both $\mathrm{MnSt}_{2}(\mathrm{~L}=\mathrm{HdAm}, \mathrm{OlAm}, \mathrm{DdTh}$, $\mathrm{OlAm} / \mathrm{DdTh}$, and StAc) and $\mathrm{Mn}_{2}(\mathrm{CO})_{10}(\mathrm{~L}=\mathrm{HdAm}, \mathrm{OlAm}$, StAc/HdAm, and StAc/OlAm) as a precursor (see Figure 6). For the sake of clarity, the ATR-IR results will be discussed in the same order as in previous subsections.
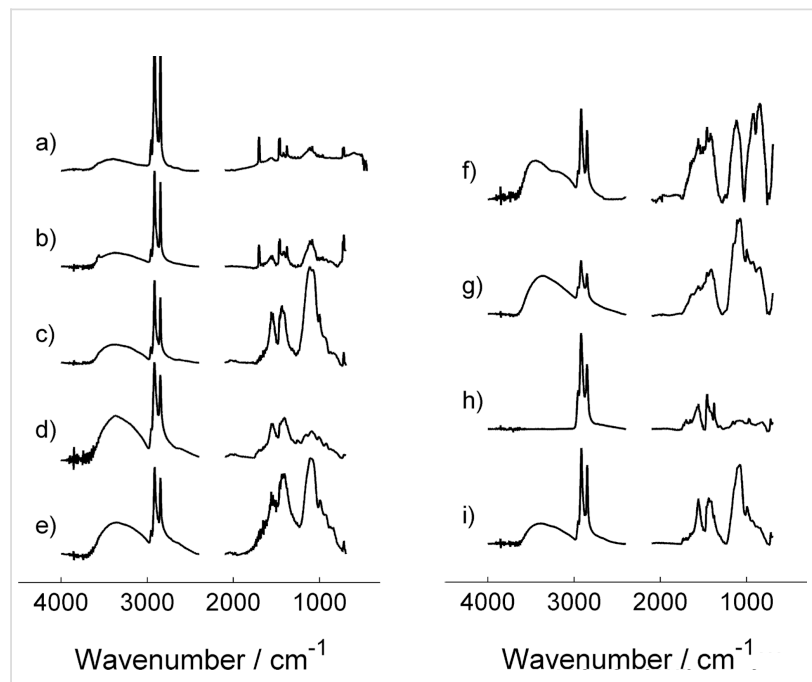

Figure 6: FTIR-ATR spectra of MnS NCs (selection of significant ranges). Left: spectra of NCs prepared using $\mathrm{MnSt}_{2}$ as a precursor with $S / M n=2$ and L/Mn = 4; L = StAc (a), DdTh (b), HdAm (c), OIAm (d), OIAm/DdTh 1:1 mixture (e). Right: spectra of NCs prepared using $\mathrm{Mn}_{2}(\mathrm{CO})_{10}$ as a precursor with $\mathrm{S} / \mathrm{Mn}=2$. Spectra $(\mathrm{f})$ and $(\mathrm{g})$ correspond to NCs prepared with amine surfactant only $(\mathrm{L} / \mathrm{Mn}=4: 1)$; $\mathrm{L}=\mathrm{HdAm}(\mathrm{f})$, OIAm (g). Spectra (h) and (i) correspond to NCs prepared using a mixture of $\mathrm{StAc}\left(\mathrm{L}_{\mathrm{acid}} / \mathrm{Mn}=2: 1\right)$ and amine surfactant $\left(\mathrm{L}_{\text {amine }} / \mathrm{Mn}=4: 1\right) ; \mathrm{L}_{\text {amine }}=\operatorname{HdAm}(\mathrm{h})$, OIAm (i).

Using $\mathrm{MnSt}_{2}$ as a precursor, both StAc and DdTh gave $\alpha-\mathrm{MnS}$ NCs. The spectra of these NCs are very similar (Figure 6a,b). The presence of both stearate and StAc is clearly discernible: two doublets $v_{\mathrm{as}}(\mathrm{C}=\mathrm{O})$ and $v_{\mathrm{s}}(\mathrm{C}=\mathrm{O})$ of carboxylate moiety are located at 1564, 1552 and $1471,1462 \mathrm{~cm}^{-1}$, respectively, and a doublet of $v(\mathrm{C}=\mathrm{O})$ of the $\mathrm{COOH}$ group is located at 1705 and $1699 \mathrm{~cm}^{-1}$. The bands located at 1462 and $1564 \mathrm{~cm}^{-1}$ closely resemble those of $\mathrm{M}^{2+}$ stearates. The occurrence of a splitting of $v(\mathrm{C}=\mathrm{O})$ bands of StAc and stearates is reminiscent of the Davydov splitting occurring in highly ordered structures such as Langmuir-Blodgett films [43]. This suggests the formation of ordered layers on the NCs surface. Notably, the presence of strongly adsorbed StAc on metal sulfides surfaces, which could not be eliminated even with several washing cycles, is in agreement with previous papers: Hironaka et al. [44] reported on StAc adsorbing more strongly on sulfides, such as FeS, rather 
than on oxides. The presence of DdTh on NCs prepared by decomposing $\mathrm{MnSt}_{2}$ with free DdTh can be ruled out by the absence of absorption bands ascribable to DdTh and by the close similarity of this spectrum to the spectrum of NCs prepared in the presence of free StAc.

The NCs synthesized by decomposing $\mathrm{MnSt}_{2}$ in the presence of an amine (yielding $\gamma$-MnS NCs) gave similar spectra, where bands corresponding to both stearate and amine can be discerned. For example, when OlAm or HdAm were used (Figure 6c,d), the presence of the corresponding amines can be inferred from the band located at $720 \mathrm{~cm}^{-1}$ (ascribable to the $\gamma_{\mathrm{w}}\left(\mathrm{NH}_{2}\right)$ wagging vibration [45]), while stearate originates two broad bands at 1551 and $1433 \mathrm{~cm}^{-1}$ (due to $v_{\mathrm{s}}(\mathrm{C}=\mathrm{O}$ ) and $v_{\mathrm{as}}(\mathrm{C}=\mathrm{O})$ of the carboxylate group). Hence, the NCs seem to be coated with both surfactants. The presence of oleylammonium or hexadecyammonium stearate salts cannot be excluded; however, there is no positive evidence, since the presence of carboxylate is not sufficient proof. Similar conclusions also apply to the spectrum of $\gamma$-MnS NCs prepared using a DdTh/OlAm mixture (Figure 6e).

We now turn to NCs prepared using $\mathrm{Mn}_{2}(\mathrm{CO})_{10}$ as a precursor. We first note that the strong $\mathrm{CO}$ stretching absorption bands of the precursor $\left(1976,2010,2045 \mathrm{~cm}^{-1}\right)$ [46] were not detectable, indicating its absence in the product. When the latter was decomposed in the presence of either HdAm or OlAm alone, $\alpha-\mathrm{MnS}$ NCs were unexpectedly obtained. These two samples (Figure 6f,g) show intense bands at 2955, 2920 and $2850 \mathrm{~cm}^{-1}$, originated by $v_{\mathrm{s} \text {,as }}(\mathrm{C}-\mathrm{H})$ vibrations of aliphatic $\mathrm{CH}_{2}$ and $\mathrm{CH}_{3}$ groups, and two complex system of bands located between 800-1200 and 1300-1700 $\mathrm{cm}^{-1}$, respectively. The latter system, even if significantly broadened, closely resembles the 1600-1300 $\mathrm{cm}^{-1}$ spectral region of pure OlAm and HdAm (1467 $\mathrm{cm}^{-1}$ : $\mathrm{C}-\mathrm{H}$ bending, $1379 \mathrm{~cm}^{-1}$ : $\mathrm{C}-\mathrm{C}$ deformation). Conversely, the $800-1200$ region is not easily attributable to any vibration of pure amines. We can conclude that these NCs are coated with the corresponding amine, as expected.

Finally, the spectra of the NCs obtained from $\mathrm{Mn}_{2}(\mathrm{CO})_{10}$ using the mixture StAc/HdAm and StAc/OlAm (Figure 6h,i) closely resemble those observed for $\gamma$-MnS NCs prepared using $\mathrm{MnSt}_{2}$ with HdAm or OlAm, respectively. This is particularly true when the $1300-1600 \mathrm{~cm}^{-1}$ spectral region is considered. The coating of the NCs prepared using the surfactant mixture thus comprises stearate and amines, and the presence of ammonium salts could not be excluded nor confirmed. It also is noteworthy that there is no clear difference between the final coating of $\alpha-\mathrm{MnS}$ and $\gamma$-MnS NCs. These spectra further confirm that it is immaterial that StAc is present as free surfactant or bound in the Mn precursor.
The $\mathrm{C}-\mathrm{H}$ stretching of vinyl protons was not observed in NC spectra, including those prepared in the presence of unsaturated compounds only (e.g., Figure 6f). This behavior has been already observed, mainly when OlAc is involved, and is probably due the disappearance of the $\mathrm{C}=\mathrm{C}$ double bond. Environmental effects leading to shifting/broadening of the vinyl $\mathrm{C}-\mathrm{H}$ stretching band can be ruled out since the latter can be observed in close-packed OlAc monolayers [47].

In summary, NCs prepared in the absence of amines show the presence of both stearic acid and stearate. Conversely, when stearic acid and an amine were present in the reaction mixture, both stearates and amines could be detected in the NC coating. This is noteworthy since carboxylic acids are usually able to displace amines from the surface of inorganic NCs, where they bind as carboxylate anion. Unfortunately, a clear relationship between adsorbed species and the polymorphism control is not readily evident.

\section{Conclusion}

In this work we have shown how the outcome of the solvothermal synthesis of $\mathrm{MnO}$ and $\mathrm{MnS}$ NCs can be controlled by varying and concentration of sulfur and surfactant and the nature of the latter. The chemical composition of the NCs (MnO vs $\mathrm{MnS}$ ) can be predicted on the basis of the $\mathrm{S} / \mathrm{Mn}$ and $\mathrm{L} / \mathrm{Mn}$ molar ratios: $\mathrm{MnS}$ is obtained when $\mathrm{S} / \mathrm{Mn}$ is larger than L/Mn. More interestingly, the polymorphism of the MnS NCs can be controlled by appropriate choice of the surfactant. $\alpha$-MnS NCs are obtained when no or a single surfactant is present, irrespective of the chemical nature of the latter. When a 1:2 mixture of carboxylic acid and amine is used, $\gamma$-MnS NCs are instead obtained.

The $\alpha$ - vs $\gamma$-MnS control does not depend on the details of the chemical structure of amine and carboxylic acid and is also independent of whether the carboxylic acid is present as a free surfactant or is contained in the manganese precursor. The peculiar role of the amine (no other surfactant in combination with carboxylic acid is able to direct the synthesis to $\gamma-\mathrm{MnS}$ NCs) seems to be related to its basicity. The ATR-IR results provided some information about the surfactants stabilizing the final NCs: those prepared in absence of amines show the presence of both stearic acid and stearate, whereas in presence of stearic acid and an amine, both stearate and amine (or alkylammonium cation) could be detected although carboxylic acids are usually stronger ligands than amines.

The chemistry occurring in the reported reactions is quite complex, especially in the formation of $\gamma-\mathrm{MnS} \mathrm{NCs}$, where both stearic acid and amine are present. As we have seen, 1-octadecene can react with sulfur at $180{ }^{\circ} \mathrm{C}$ to produce $\mathrm{H}_{2} \mathrm{~S}$ but 
it was also shown that long chain alkylamines react with sulfur following several two-step pathways, all of which produce $\mathrm{H}_{2} \mathrm{~S}$ at $130{ }^{\circ} \mathrm{C}$ [48]. To add to the complexity, carboxylic acids may react with both alkylamines and $\mathrm{H}_{2} \mathrm{~S}$. It is clearly difficult to understand the peculiar chemistry leading to $\gamma$-MnS NCs as many reacting species are simultaneously present. However, two brief considerations can be made about the specificity of the amine/carboxylic acid mixture. First, we found that the carboxylic acid is equally effective both as a carboxylate ligand in the precursor and free acid. This could be explained by the formation of a manganese stearate intermediate when $\mathrm{Mn}_{2}(\mathrm{CO})_{10}$ is heated in the presence of StAc, similar to the case of $\mathrm{Fe}(\mathrm{CO})_{5}$ and oleic acid that give an iron oleate intermediate in the synthesis of iron oxide NCs [49]. Second, the formation of $\gamma$-MnS NCs seems to be related to the basicity of amines but it only occurs when a carboxylic acid is also present. This suggests that monoalkylammonium carboxylate salts $\left(\mathrm{R}^{1}-\mathrm{NH}_{3}{ }^{+}\right.$ $\mathrm{R}^{2}-\mathrm{COO}^{-}$), which were reported to form in an apolar solvent such as cyclohexane [45], may play a role in the polymorphism control. Unfortunately, ATR-IR measurements could not confirm (nor exclude) the presence of long chain ammonium stearates on the final NCs since their spectrum is very close to that of a mixture of stearate and amine molecules chemisorbed on the MnS surface.

\section{Experimental}

Materials

Manganese(II) chloride (reagent grade, $\geq 99 \%$ ), tetramethylammonium hydroxide pentahydrate, sulfur powder $(99.98 \%)$, dimanganese decacarbonyl (98\%), oleic acid (90\%), stearic acid (99\%), oleylamine (technical grade, 70\%), dodecylamine (98\%), hexadecylamine (90\%), octadecylamine ( $\geq 99 \%)$, dodecanthiol (98\%), oleylalcohol ( $\geq 85 \%$ ), tryoctylphosphine (97\%), and octadecene (purum, $\geq 95.0 \%$ ) were purchased from SigmaAldrich. Stearic acid (99\%) was purchased from Calbiochem. All chemicals were used as received without further purification.

\section{Synthesis of manganese(II) distearate}

Manganese distearate $\left(\mathrm{MnSt}_{2}\right)$ was synthesized by a modification of the procedure reported in [23]. In a three-necked flask, a clear solution of of tetramethylammonium hydroxide pentahydrate $(4.55 \mathrm{~g}, 50 \mathrm{mmol})$ and stearic acid $(14.2 \mathrm{~g}, 50 \mathrm{mmol})$ in methanol $(175 \mathrm{~mL})$ was prepared and stirred under argon for $1 \mathrm{~h}$ at room temperature. Then, the mixture was cooled to $0{ }^{\circ} \mathrm{C}$ using an ice bath and a solution of anhydrous $\mathrm{MnCl}_{2}(3.15 \mathrm{~g}$, $25 \mathrm{mmol})$ in methanol $(100 \mathrm{~mL})$ was slowly added. A white precipitate immediately formed, which was collected by filtration using a Buchner funnel previously cooled to $-20{ }^{\circ} \mathrm{C}$ and washed with cold methanol ( 3 times, $10 \mathrm{~mL}$ ). Drying under vacuum ( $p \approx 10^{-2}$ torr) for $6 \mathrm{~h}$ gave $\mathrm{MnSt}_{2}$ as a white powder.
The average yield was $\approx 50 \%$. IR $(\mathrm{KBr}), v\left(\mathrm{~cm}^{-1}\right): 2955,2917$, 2849, 1552, 1468, 1440, 1320, 1113, 949, 721.

\section{Synthesis of manganese(II) monooleate}

Manganese monooleate hydroxide $(\mathrm{Mn}(\mathrm{Ol})(\mathrm{OH}))$ was synthesized following the procedure reported in [23].

\section{Synthesis of $\operatorname{MnX}(X=S, O)$ NCs}

The procedure to prepare MnX NCs is described with reference to the $\mathrm{S} / \mathrm{L} / \mathrm{Mn}=2: 4: 1$ case. In a two-necked flask, a solution of manganese(II) distearate $(0.26 \mathrm{mmol}, 0.25 \mathrm{M})$, sulfur $(0.52 \mathrm{mmol}, 0.50 \mathrm{M})$, and stearic acid $(1.04 \mathrm{mmol}, 1.00 \mathrm{M})$ in 1 -octadecene was prepared. The solution was heated to $320^{\circ} \mathrm{C}$ (heating rate $=10^{\circ} \mathrm{C} / \mathrm{min}$ ) under nitrogen and magnetic stirring. After $1 \mathrm{~h}$, the reaction mixture was cooled to room temperature. The NCs were precipitated by adding the reaction crude with a five-fold excess of ethanol and then collected by centrifugation (6000 rpm, $10 \mathrm{~min}$ ). Next, the NCs were washed with ethanol and collected by centrifugation (three times) and then washed with acetone and collected by centrifugation (three times). The precipitate was finally dispersed in hexane $(5-10 \mathrm{~mL})$ by sonication (ultrasonic bath, $1 \mathrm{~h}, \mathrm{RT}$ ). The obtained NC dispersions are stable for several months.

Several variants of this procedure were carried out by changing:

1. the manganese precursor (manganese distearate $\left(\mathrm{MnSt}_{2}\right)$ or $\left.\mathrm{Mn}_{2}(\mathrm{CO})_{10}\right)$;

2. the $\mathrm{S} / \mathrm{Mn}$ molar ratio (from 0:1 to $4: 1$ );

3 . the free surfactant $(\mathrm{L}=$ stearic acid (StAc), hexadecylamine (HdAm), dodecylamine (DdAm), octadecylamine (OdAm), oleylamine (OlAm), oleylalcohol (OlAl), or dodecanethiol (DdTh)); and

4. the $\mathrm{L} / \mathrm{Mn}$ molar ratio (from $0: 1$ to $4: 1$ ).

\section{Characterization}

Conventional transmission electron microscopy (TEM) images (medium resolution), high resolution TEM (HRTEM) images and electron diffraction (ED) patterns were recorded by a Zeiss LIBRA 200FE-HR TEM. The samples for microscopy were prepared by evaporating a drop of the nanocrystal dispersion in hexane on a carbon-coated TEM grid. ATR-FTIR spectra ( $4 \mathrm{~cm}^{-1}$ resolution, 50 scans) were collected using an ATR platform (Golden Gate, Specac) mounted in a spectrophotometer (FTS-40, Biorad) equipped with a $\mathrm{KBr}$ beam splitter and a MCT detector operating between 400 and $4000 \mathrm{~cm}^{-1}$. For ATRFTIR spectroscopy, the purified NCs were further washed $(2 \times)$ with a large excess of acetone and then collected by centrifugation (10000 rpm, $10 \mathrm{~min})$. NCs with $\mathrm{L}=$ hexadecylamine were washed with $\mathrm{CHCl}_{3}$ to effectively remove the residual surfactant. 


\section{Supporting Information}

\section{Supporting Information File 1}

Additional TEM images and ED patterns of $\mathrm{MnO}$ and $\mathrm{MnS}$ nanocrystals.

[http://www.beilstein-journals.org/bjnano/content/ supplementary/2190-4286-6-238-S1.pdf]

\section{Acknowledgements}

The authors gratefully acknowledge financial support by Fondazione Cariplo under grant 2014-1865 (New class of electrodes for photoelectrochemical water splitting), Regione Lombardia in the framework of the Second Agreement with CNR (RSPPTECH project), and the Italian MIUR under grant FIRB RBAP115AYN (Oxides at the nanoscale: multifunctionality and applications).

\section{References}

1. Lokhande, C. D.; Ennaoui, A.; Patil, P. S.; Giersig, M.; Diesner, K.; Tributsch, H. Thin Solid Films 1998, 330, 70-75. doi:10.1016/S0040-6090(98)00500-8

2. Papp, G. History of minerals, rocks and fossil resins discovered in the Carpathian region; Hungarian Natural History Museum: Budapest, Hungary, 2004.

3. Eriksson, L.; Kalinowski, M. P. Acta Crystallogr., Sect. E: Struct. Rep. Online 2001, E57, i92-i93. doi:10.1107/S1600536801016051

4. Ma, C.; Beckett, J. R.; Rossman, G. R. Am. Mineral. 2012, 97, 2056-2059. doi:10.2138/am.2012.4286

5. Corliss, L.; Elliott, N.; Hastings, J. Phys. Rev. 1956, 104, 924-928. doi:10.1103/PhysRev.104.924

6. Danielian, A.; Stevens, K. W. H. Proc. Phys. Soc., London 1961, 77, 124-128. doi:10.1088/0370-1328/77/1/315

7. Viswanath, R.; Naik, H. S. B.; Kumar, G. S. Y.; Kumar, P. N. P.; Harish, K. N.; Prabhakara, M. C. Spectrochim. Acta, Part A 2014, 125, 222-227. doi:10.1016/j.saa.2014.01.022

8. Zhang, X. V.; Martin, S. T.; Friend, C. M.; Schoonen, M. A. A.; Holland, H. D. J. Am. Chem. Soc. 2004, 126, 11247-11253. doi:10.1021/ja0476415

9. Beltran-Huarac, J.; Resto, O.; Carpena-Nuñez, J.; Jadwisienczak, W. M.; Fonseca, L. F.; Weiner, B. R.; Morell, G. ACS Appl. Mater. Interfaces 2014, 6, 1180-1186. doi:10.1021/am404746k

10. Tang, Y.; Chen, T.; Yu, S. Chem. Commun. 2015, 51, 9018-9021. doi:10.1039/C5CC01700A

11. Ge, J.; Li, Y. Chem. Commun. 2003, 2498-2499. doi:10.1039/b307452h

12. Ge, J.-P.; Wang, J.; Zhang, H.-X.; Li, Y.-D. Chem. - Eur. J. 2004, 10, 3525-3530. doi:10.1002/chem.200400008

13. Kim, D. S.; Lee, J. Y.; Na, C. W.; Yoon, S. W.; Kim, S. Y.; Park, J.; Jo, Y.; Jung, M.-H. J. Phys. Chem. B 2006, 110, 18262-18266. doi:10.1021/jp063965z

14. An, C.; Tang, K.; Liu, X.; Li, F.; Zhou, G.; Qian, Y. J. Cryst. Growth 2003, 252, 575-580. doi:10.1016/S0022-0248(03)01001-7
15. Gui, Y.; Qian, L.; Qian, X. Mater. Chem. Phys. 2011, 125, 698-703. doi:10.1016/j.matchemphys.2010.09.071

16. Mu, J.; Gu, Z.; Wang, L.; Zhang, Z.; Sun, H.; Kang, S.-Z. J. Nanopart. Res. 2008, 10, 197-201. doi:10.1007/s11051-007-9216-8

17. Moloto, N.; Moloto, M. J.; Kalenga, M.; Govindraju, S.; Airo, M. Opt. Mater. 2013, 36, 31-35. doi:10.1016/j.optmat.2013.06.023

18. Kan, S.; Felner, I.; Banin, U. Isr. J. Chem. 2001, 41, 55-61. doi:10.1560/1FB3-1PF4-72JQ-0AQC

19. Jun, Y.-w.; Jung, Y.-y.; Cheon, J. J. Am. Chem. Soc. 2002, 124, 615-619. doi:10.1021/ja016887w

20. Pradhan, N.; Katz, B.; Efrima, S. J. Phys. Chem. B 2003, 107, 13843-13854. doi:10.1021/jp035795I

21. Tian, L.; Yep, L. Y.; Ong, T. T.; Yi, J.; Ding, J.; Vittal, J. J. Cryst. Growth Des. 2009, 9, 352-357. doi:10.1021/cg800536w

22. Wang, D.-S.; Zheng, W.; Hao, C.-H.; Peng, Q.; Li, Y.-D. Chem. - Eur. J. 2009, 15, 1870-1875. doi:10.1002/chem.200801815

23. Puglisi, A.; Mondini, S.; Cenedese, S.; Ferretti, A. M.; Santo, N.; Ponti, A. Chem. Mater. 2010, 22, 2804-2813. doi:10.1021/cm903735e

24. Tian, Q.; Tang, M.; Jiang, F.; Liu, Y.; Wu, J.; Zou, R.; Sun, Y.; Chen, Z.; Li, R.; Hu, J. Chem. Commun. 2011, 47, 8100-8102. doi:10.1039/c1cc11621e

25. Yang, X.; Wang, Y.; Sui, Y.; Huang, X.; Cui, T.; Wang, C.; Liu, B.; Zou, G.; Zou, B. Langmuir 2012, 28, 17811-17816. doi:10.1021/la304228w

26. Yang, X.; Wang, Y.; Wang, K.; Sui, Y.; Zhang, M.; Li, B.; Ma, Y.; Liu, B.; Zou, G.; Zou, B. J. Phys. Chem. C 2012, 116, 3292-3297. doi:10.1021/jp209591r

27. Peng, L.; Shen, S.; Zhang, Y.; Xu, H.; Wang, Q. J. Colloid Interface Sci. 2012, 377, 13-17. doi:10.1016/j.jcis.2012.03.052

28. Joo, J.; Na, H. B.; Yu, T.; Yu, J. H.; Kim, Y. W.; Wu, F.; Zhang, J. Z.; Hyeon, T. J. Am. Chem. Soc. 2003, 125, 11100-11105. doi:10.1021/ja0357902

29. Zhang, H.; Hyun, B.-R.; Wise, F. W.; Robinson, R. D. Nano Lett. 2012, 12, 5856-5860. doi:10.1021/nl303207s

30. Choi, S.-H.; An, K.; Kim, E.-G.; Yu, J. H.; Kim, J. H.; Hyeon, T. Adv. Funct. Mater. 2009, 19, 1645-1649. doi:10.1002/adfm.200800832

31. Hÿtch, M. J.; Snoeck, E.; Kilaas, R. Ultramicroscopy 1998, 74, 131-146. doi:10.1016/S0304-3991(98)00035-7

32. Shannon, R. D. Acta Crystallogr., Sect. A: Found. Adv. 1976, A32, 751-767. doi:10.1107/S0567739476001551

33. Meyer, B. Chem. Rev. 1976, 76, 367-388. doi:10.1021/cr60301a003

34. Li, Z.; Ji, Y.; Xie, R.; Grisham, S. Y.; Peng, X. J. Am. Chem. Soc. 2011, 133, 17248-17256. doi:10.1021/ja204538f

35. Cronyn, M. W.; Jiu, J. J. Am. Chem. Soc. 1952, 74, 4726. doi:10.1021/ja01138a527

36. Roy, P. K.; Surekha, P.; Raman, R.; Rajagopal, C. Polym. Degrad. Stab. 2009, 94, 1033-1039. doi:10.1016/j.polymdegradstab.2009.04.025

37. Pan, D.; Senpan, A.; Caruthers, S. D.; Williams, T. A.; Scott, M. J.; Gaffney, P. J.; Wickline, S. A.; Lanza, G. M. Chem. Commun. 2009, 3234-3236. doi:10.1039/b902875g

38. Laurence, C.; Gal, J.-F. Lewis Basicity and Affinity Scales: Data and Measurement; John Wiley \& Sons: Hoboken, NJ, U.S.A., 2010.

39. Mayr, H.; Ofial, A. R. J. Phys. Org. Chem. 2008, 21, 584-595. doi:10.1002/poc.1325

40. Mondini, S.; Cenedese, S.; Marinoni, G.; Molteni, G.; Santo, N.; Bianchi, C. L.; Ponti, A. J. Colloid Interface Sci. 2008, 322, 173-179. doi:10.1016/j.jcis.2008.03.008

41. van Embden, J.; Chesman, A. S. R.; Jasieniak, J. J. Chem. Mater. 2015, 27, 2246-2285. doi:10.1021/cm5028964 
42. Mourdikoudis, S.; Liz-Marzan, L. M. Chem. Mater. 2013, 25, 1465-1476. doi:10.1021/cm4000476

43. Baran, J.; Marchewka, M. K.; Ratajczak, H.; Borovikov, A. Yu.; Byckov, V. N.; Naumovets, A. G.; Podzelinsky, A. V.;

Puchkovskaya, G. A.; Styopkin, V. I. Thin Solid Films 1995, 254 , 229-239. doi:10.1016/0040-6090(94)06253-H

44. Hironaka, S.; Yahagi, Y.; Sakurai, T. ASLE Trans. 1978, 21, 231-235. doi:10.1080/05698197808982879

45. Klokkenburg, M.; Hilhorst, J.; Ernè, B. H. Vib. Spectrosc. 2007, 43, 243-248. doi:10.1016/j.vibspec.2006.09.008

46. Tjahjono, M.; Allian, A. D.; Garland, M. Dalton Trans. 2006, 1505-1516. doi:10.1039/b515298d

47. Dluhy, R. A.; Cornell, D. G. J. Phys. Chem. 1985, 89, 3195-3197. doi:10.1021/j100261a006

48. Thomson, J. W.; Nagashima, K.; Macdonald, P. M.; Ozin, G. A. J. Am. Chem. Soc. 2011, 133, 5036-5041. doi:10.1021/ja1109997

49. Hyeon, T.; Lee, S. S.; Park, J.; Chung, Y.; Na, H. B. J. Am. Chem. Soc. 2001, 123, 12798-12801. doi:10.1021/ja016812s

\section{License and Terms}

This is an Open Access article under the terms of the Creative Commons Attribution License (http://creativecommons.org/licenses/by/2.0), which permits unrestricted use, distribution, and reproduction in any medium, provided the original work is properly cited.

The license is subject to the Beilstein Journal of

Nanotechnology terms and conditions:

(http://www.beilstein-journals.org/bjnano)

The definitive version of this article is the electronic one which can be found at: doi:10.3762/bjnano.6.238 